\title{
A SiGe-Source Doping-Less Double-Gate Tunnel FET: Design and Analysis Based on Charge Plasma Technique with Enhanced Performance
}

\section{Varun Mishra ( $\nabla$ rel1605@mnnit.ac.in )}

Graphic Era Deemed to be University https://orcid.org/0000-0002-9264-9338

\section{Yogesh Kumar Verma}

Lovely Professional University

\section{Santosh Kumar Gupta}

MNNIT Allahabad: Motilal Nehru National Institute of Technology

\section{Vikas Rathi}

Graphic Era Deemed to be University

\section{Research Article}

Keywords: Tunnel field effect transistor (TFET), charge plasma, doping-less, band-to-band tunneling (BTBT), low energy bandgap material

Posted Date: February 24th, 2021

DOl: https://doi.org/10.21203/rs.3.rs-229785/v1

License: (c) (i) This work is licensed under a Creative Commons Attribution 4.0 International License. Read Full License

Version of Record: A version of this preprint was published at Silicon on March 2nd, 2021. See the published version at https://doi.org/10.1007/s12633-021-01030-6. 


\section{Abstract}

In this article, a distinctive charge plasma (CP) technique is employed to design two doping-less dual gate tunnel field effect transistors (DL-DG-TFETs) with $\mathrm{Si}_{0.5} \mathrm{Ge}_{0.5}$ and $\mathrm{Si}$ as source material. The CP methodology resolves the issues of random doping fluctuation and doping activation. The analog and RF performance has been investigated for both the proposed devices i.e. $\mathrm{Si}_{0.5} \mathrm{Ge}_{0.5}$ source DL-DG-TFET and Si-source DL-DG-TFET in terms of drive current, transconductance, cut-off frequency. In addition, the linearity and distortion analysis has been carried out for both the proposed devices with respect to higher order transconductance $\left(\mathrm{g}_{\mathrm{m} 2}\right.$ and $\left.\mathrm{g}_{\mathrm{m} 3}\right)$, VIP2, IMD3, and HD2. The $\mathrm{Si}_{0.5} \mathrm{Ge}_{0.5}$ source DL-DG-TFET has better performance characteristics and reliability in compare to Si-source DL-DG-TFET owing to low energy bandgap material and higher mobility. The switching ratio obtained for $\mathrm{Si}_{0.5} \mathrm{Ge}_{0.5}$ source DL-DGTFET is order of $5 \times 10^{14}$ that makes it a suitable contender for low power applications.

\section{Introduction}

In the coming decade, Moore's law will be relevant only by scaling down the power consumption along with process size. Miniaturizing the size of MOSFETs give rise to various issues related to leakage power, fabrication, subthreshold swing (SS), gate induced drain lowering (GIDL), and several other second order effects [1], [2].

These complications enforce to search for the novel devices, whose working philosophy is other than thermionic emission. In this aspect, Tunnel FET has come up as a strong candidate to outplay the conventional MOSFETs. TFETs exhibits low leakage current, SS below $60 \mathrm{mV} /$ decade, and minimized SCEs due to its in-built tunneling barrier. The advantage of TFETs over MOSFETs is its better performance below $1 \mathrm{~V}$.

TFETs employ band to band tunneling; the OFF-state current obtained is low due to large tunneling width for smaller biasing which prohibits the tunneling of charge carriers. The constraint of TFETs till date is low ON current and high ambipolar current [3].

The ambipolar effect can be minimized by employing lightly doped drain region and gate-drain underlap [4]. The tunneling path increases at channel-drain junction by decreasing the doping concentration of drain that suppresses the ambipolar current. To increase the drain current, various methods have been reported such as high-k dielectric engineering [5], L-shaped gate [6], triple material double gate [7], heterojunction [8], hetero dielectric [9], junctionless [10]. The inclusion of ferroelectric material as gate insulator also increases the tunneling rate that further increase the drive current and suppresses the subthreshold swing [11][12].

The double gate methodology provides better electrostatics restriction over the channel that enhances the device characteristics by increasing the inversion charge carriers. Incorporation of low band gap materials such as $\mathrm{Si}_{x} \mathrm{Ge}_{1-x}, \mathrm{Ge}$, or InAs at source side minimizes the tunneling path thereby increasing the 
ON-state current [13]. Another issue is of uniform doping that originates during fabrication process; due to scaling down the doping becomes very difficult and costly for the formation of p-n junction. The junction less transistors (JLTs) were introduced by Tyndall's group that does not has any metallurgical junction and has uniform high doping throughout the source, channel and drain regions [14]. The JLTs suffered from random dopant fluctuations (RDF) [15] that severely deteriorates the characteristics of the device.

In order to address the RDF effects, charge plasma methodology has been suggested by Rajasekharan et al [16] to enhance the device performance. Charge plasma techniques is a high temperature process that employs electrodes with suitable work functions to induce doped drain and source regions on an undoped substrate i.e. doping-less (DL) [17].

In this work, charge plasma technique along with low band gap material at source is employed to improve the performance of double gate tunnel FET (DL-DG-TFET). The $\mathrm{Si}_{0.5} \mathrm{Ge}_{0.5}$ and $\mathrm{Si}$ based source doping-less DG-TFETs are compared in accordance with analog, RF, linearity and distortion performance metrics. Furthermore, the linearity and distortion analysis of the two devices i.e. $\mathrm{Si}_{0.5} \mathrm{Ge}_{0.5}$ source DL-DGTFET and Si source DL-DG-TFET has been carried out. The aim is to obtain the improved performance of the device in terms of ON-current, subthreshold swing, linearity, and distortion.

\section{li. Device Dimensions And Models}

The 2-D structures of the intended devices, DL-DG-TFET with $\mathrm{Si}_{0.5} \mathrm{Ge}_{0.5}$ and $\mathrm{Si}$ source has been presented in figure 1(a) and (b) respectively. The undoped source, drain, and channel regions of the proposed device are formed on an intrinsically doped Si film with $n_{i}=10^{15} \mathrm{~cm}^{-3}$. The thickness of undoped Si body is $5 \mathrm{~nm}$. Charge plasma methodology has been employed in order to induce doping in the source and drain regions of the proposed devices. Charge plasma technique is applicable only when the Debye length is greater than channel thickness.

The drain region material of both the proposed devices are same to obtain the low OFF-state current, however, the source material in one of the device is a low band gap material i.e. $\mathrm{Si}_{0.5} \mathrm{Ge}_{0.5}$, to achieve high drive current.

The two gates are connected with the device namely control gate and fixed gate. Control gate is present above the channel region to control the flow of majority charge carriers. However, the fixed gate is located above the source region to create the p-type doping in source. Control and fixed gate are isolated by spacer $\left(\mathrm{SiO}_{2}\right)$ of length $2 \mathrm{~nm}$. The work function of fixed gate and source electrode is selected to be 5.93 $\mathrm{eV}$ (Platinum) to induce p-type doping (accumulation of positive charges) in source region. Furthermore, the work function of drain electrode is $3.9 \mathrm{eV}$ (Hafnium) to induce n-type doping in drain region. The work function of control gate is $4.2 \mathrm{eV}$ (Aluminium). The insulator $\left(\mathrm{SiO}_{2}\right)$ thickness for both the device is $2 \mathrm{~nm}$. For optimum performance, the mole fraction of SiGe is taken as 0.5 [17]. The channel length for both the proposed devices is $22 \mathrm{~nm}$; drain and source regions is of $20 \mathrm{~nm}$ each. 
Numerical calculations has been carried out by using 2D device simulator, Silvaco TCAD, for both the proposed devices. The models used during the numerical simulation are Shockley-Read Hall recombination model, Lombardi mobility model, quantum model, non-local BTBT model, and phononassisted tunneling model. Non local model inspects the spatial variation of energy bands. Quantum model was employed as the thickness of channel is $5 \mathrm{~nm}$.

\section{lii. Results And Discussion}

In this work, the performance analysis has been carried out for the proposed devices DL-DG-TFET with Si and $\mathrm{Si}_{0.5} \mathrm{Ge}_{0.5}$ source. The numerical calculation models utilized for the analysis are calibrated against the previously published result [18]. In order to fine-tune the Kane's tunneling model, its tunneling masses are tuned from their standard values to a adjusted value, i.e. me.tunnel $=0.272 m_{0}$ and $m$ h.tunnel $=$ $0.54 m_{0}$, where $m_{0}$ is the electron rest mass. Figure 2 shows the model validation, all the dimensions and doping parameters are kept identical to that of [18].

The energy band diagram variation along the channel in OFF and ON-state for both the proposed devices is shown in Fig. 3(a) and $3(\mathrm{~b})$, respectively. In OFF-state the gate voltage $\left(\mathrm{V}_{\mathrm{GS}}=0 \mathrm{~V}\right)$ is zero, and drain voltage $\left(V_{D S}=1 \mathrm{~V}\right)$ is $1 \mathrm{~V}$. However, in the $O N$-state the $V_{G S}=1 \mathrm{~V}$, and $V_{D S}=1 \mathrm{~V}$. The incorporation of low band gap material $\left(\mathrm{Si}_{0.5} \mathrm{Ge}_{0.5}\right)$ at source side reduces the tunneling path, as it can be observed from Fig. $3(b)$. Consequently, the reduction in tunneling path minimizes the tunneling resistance and enhances the tunneling of charge carriers. The band gap of $\mathrm{Si}_{0.5} \mathrm{Ge}_{0.5}$ is $0.94 \mathrm{eV}$, which is smaller than $\mathrm{Si}$, minimizes the tunneling path that additionally increases the tunneling rate of charge carriers. The WKB tunneling probability approximation of $\mathrm{Si}_{0.5} \mathrm{Ge}_{0.5}$ source DL-DG-TFET is given below:

$$
\begin{aligned}
& T(E)=\exp \left(-\frac{4 \lambda \sqrt{2 m^{*}} E_{g}^{1.5}}{3 q h\left(E_{g}+\Delta \phi\right)}\right) \\
& \lambda=\sqrt{\frac{\varepsilon_{S i} t_{S i} t_{o x}}{\varepsilon_{o x}}}
\end{aligned}
$$

where $\lambda$ is screening length, $E_{g}$ is energy bandgap, $\Delta \phi$ is potential difference between source valence band and channel conduction band, and $m^{*}$ is effective mass. It can be observed from (1) that tunneling probability increases with low energy bandgap and low effective mass. Therefore, the tunneling probability is high for $\mathrm{Si}_{0.5} \mathrm{Ge}_{0.5}$ source DL-DG-TFET that can be reflected from Fig. 4 with increased BTBT rate, as the $\mathrm{Si}_{0.5} \mathrm{Ge}_{0.5}$ has lower energy band gap in contrast to $\mathrm{Si}$.

Figure 5 visualizes the variation of surface potential along the channel for both the proposed devices. In addition, the enhanced tunneling rate of $\mathrm{Si}_{0.5} \mathrm{Ge}_{0.5}$ source DL-DG-TFET reflects higher surface potential. The electron concentration for the proposed devices DL-DG-TFET with $\mathrm{Si}$ and $\mathrm{Si}_{0.5} \mathrm{Ge}_{0.5}$ source has been 
shown in Fig. 6. It can be observed from Fig. 6 that the electron concentration for $\mathrm{Si}_{0.5} \mathrm{Ge}_{0.5}$-source based device is higher in contrast to Si-source. This fashion remains same until the graph reaches the drain region; further the electron concentration becomes equal for both the intended structures as the drain material is identical.

Figure 7 represents the transfer characteristics for both the proposed devices. The ON-current of $\mathrm{Si}_{0.5} \mathrm{Ge}_{0.5}$-source based device is higher in contrast to Si-source. However, OFF-current of both the devices remains same; this leads to enhance the switching ratio ( $\left.\mathrm{I}_{\mathrm{ON}} / \mathrm{I}_{\mathrm{OFF}}\right)$ for $\mathrm{Si}_{0.5} \mathrm{Ge}_{0.5}$-source based device. The drive current depends on the tunnel resistance and channel resistance. However, the tunneling width of $\mathrm{Si}_{0.5} \mathrm{Ge}_{0.5}$-source is smaller in contrast to Si-source based device, which is reflected in Fig. 3(b). Tunneling width totally depends on the tunneling resistance that is caused by proper gate to source voltage. Thus, the smaller tunneling width leads to higher drive current for $\mathrm{Si}_{0.5} \mathrm{Ge}_{0.5}$-source in compare to Si-source. The drive current and switching ratio obtained for $\mathrm{Si}_{0.5} \mathrm{Ge}_{0.5}$ source based device are $71.4 \mu \mathrm{A} / \mu \mathrm{m}$ and order of $5 \times 10^{14}$, respectively.

The transconductance $\left(\mathrm{g}_{\mathrm{m}}\right)$ variation for both the proposed devices is shown in Fig. 8. Transconductance is the significant device parameter, it evaluates the current driving potentiality of the device and is calculated as $\partial I_{D} / \partial V_{G S}$ keeping $V_{D S}$ constant. It is evident from the Fig. 7 that since $I_{D}$ has higher value for $\mathrm{Si}_{0.5} \mathrm{Ge}_{0.5}$-source based device, therefore, $\mathrm{g}_{\mathrm{m}}$ will certainly be high due to direct dependence of $\mathrm{g}_{\mathrm{m}}$ with respect to drain current.

Figure 9(a) and 9(b) presents the graph of total gate capacitance and cut-off frequency, respectively, against $\mathrm{V}_{\mathrm{GS}}$ for both the proposed structures. Total gate capacitance $\left(\mathrm{C}_{\mathrm{GG}}\right)$ is calculated by the summation of gate-to-source capacitance $\left(\mathrm{C}_{G S}\right)$ and gate-to-drain capacitance $\left(\mathrm{C}_{\mathrm{GD}}\right)$. From figure 6 , it is evident that the electron concentration for $\mathrm{Si}_{0.5} \mathrm{Ge}_{0.5}$-source based device is higher; this leads to increase in total gate capacitance of the device because of increase in gate-to-source capacitance. Therefore, $\mathrm{C}_{\mathrm{GG}}$ for $\mathrm{Si}_{0.5} \mathrm{Ge}_{0.5}$-source based device has higher value in compare to Si-source. Cut-off frequency reflects the maximal frequency that can be escalated by a discrete device and can be expressed as:

$$
f_{T}=\frac{g_{m}}{2 \pi C_{G G}}
$$

The direct dependence of cut-off frequency with transconductance confirms that higher value of $g_{m}$ will certainly have higher value of $f_{T}$. Hence, it has been observed from Fig. $9(b)$ that cut-off frequency of $\mathrm{Si}_{0.5} \mathrm{Ge}_{0.5}$-source based device is higher as its drain current and transconductance has higher value in contrast to Si-source based device.

The gain-bandwidth product (GBP) is an important metric for RF analysis and is expressed as: 
$G B P=\frac{g_{m}}{2 \pi \times 10^{*} C_{G D}}$

GBP has direct dependence on ratio of transconductance and parasitic capacitance. Figure 10 shows $G B P$ variation with respect to $V_{G S}$ for both the proposed structures. The GBP graph increases with $V_{G S}$ due to increase in transconductance and reduction in parasitic capacitance. Higher value of transconductance for $\mathrm{Si}_{0.5} \mathrm{Ge}_{0.5}$-source in compare to Si-source, results in higher value of its $\mathrm{GBP}$, as can be observed from Fig. 10. Transconductance frequency product (TFP) is another important figure of merit (FOM) for RF performance, which is expressed as:

$$
T F P=\left(\frac{g_{m}}{I_{D}}\right) \times f_{T}
$$

Figure 11 presents the TFP variation with respect to $V_{G S}$ for both the proposed structures. TFP shows the compromise between power and bandwidth [19]. It has been observed from Fig. 11 that TFP of $\mathrm{Si}_{0.5} \mathrm{Ge}_{0.5^{-}}$ source is higher in contrast to Si-source based device due to higher value of $g_{m}$ and $f_{T}$.

The linearity parameters has been evaluated in order to analyse the device for distortion free output. In order to improve linearity, the transconductance of the device should be invariant with regard to $V_{G S}[20]$. Unfortunately, the transconductance of TFET varies with gate voltage that depicts the non-linear characteristics of TFET. The higher order transconductance $\left(g_{m 2}\right.$ and $\left.g_{m 3}\right)$ are shown in Fig. 12 for both the proposed devices that depicts non-linear characteristics of device. The main reason of non-linearity is the involvement of higher order transconductance with the fundamental frequency. In contrast to $\mathrm{g}_{\mathrm{m} 2}, \mathrm{~g}_{\mathrm{m} 3}$ is more related in evaluating the non-linearity of the device. The balanced techniques can be employed to minimize the even harmonics, however $\mathrm{g}_{\mathrm{m} 3}$ is not controllable. In order to attain the least distortion, the magnitude of both high order transconductance $\left(g_{m 2}\right.$ and $\left.g_{m 3}\right)$ should be low. The $g_{m 2}$ and $g_{m 3}$ increases for $\mathrm{Si}_{0.5} \mathrm{Ge}_{0.5}$-source based device in compare to Si-source that can be viewed from Fig. 12, therefore the linearity of the device is enhanced for latter case.

Other FOMs such as VIP2 and IMD3 have been plotted with respect to $\mathrm{V}_{\mathrm{GS}}$ in Fig. 13 (a) and 13 (b) respectively. VIP2 is an extrapolated input voltage for which fundamental and second harmonic voltage are equal. IMD3 is the third order intermodulation distortion that depicts the extrapolated current at which the fundamental and third order intermodulation harmonic currents are equal. In order to attain high linearity and low distortion, higher values of VIP2 and lower values of IMD3 are required. Fig. 13 (a) presents the higher values of VIP2 for $\mathrm{Si}_{0.5} \mathrm{Ge}_{0.5}$-source that depicts the improved linearity in contrast to Si-source. The lower values of IMD3, as shown in Fig. $13(\mathrm{~b})$, for $\mathrm{Si}_{0.5} \mathrm{Ge}_{0.5}$-source confirms to have low internal noise in contrast to Si-source based device.

The second-order harmonic distortion (HD2) is a critical parameter of distortion. In order to minimize the Loading [MathJax]/jax/output/CommonHTML/fonts/TeX/fontdata.js as possible. Figure 14 presents the graph of 
$\mathrm{HD} 2$ against $\mathrm{V}_{\mathrm{GS}}$ for both the proposed structures, it can be depicted from the graph that for $\mathrm{Si}_{0.5} \mathrm{Ge}_{0.5^{-}}$ source, the value of HD2 is lower in compare to Si-source. Hence, it can be summarised that $\mathrm{Si}_{0.5} \mathrm{Ge}_{0.5^{-}}$ source based TFET has better linearity, reliability and reduced distortion then Si-source based TFET.

\section{Iv. Conclusion}

In this article, $\mathrm{CP}$ based technique has been introduced to design the doping-less dual gate structure with $\mathrm{Si}$ and $\mathrm{Si}_{0.5} \mathrm{Ge}_{0.5}$ source. The analog, $\mathrm{RF}$, linearity and distortion analysis has been carried out for both the proposed structures. The $\mathrm{Si}_{0.5} \mathrm{Ge}_{0.5}$ source based DL-DG-TFET has better characteristics in compare to Sisource based DL-DG-TFET due to assimilation of a low bandgap material in the source region. The drive current and switching ratio obtained for $\mathrm{Si}_{0.5} \mathrm{Ge}_{0.5}$ source DL-DG-TFET are $71.4 \mu \mathrm{A} / \mu \mathrm{m}$ and order of $5 \times 10^{14}$, respectively. The improved linearity and distortion metrics for $\mathrm{Si}_{0.5} \mathrm{Ge}_{0.5}$ source DL-DG-TFET in compare to Si-source DL-DG-TFET makes it a worthy contender for low power applications.

\section{Declarations}

Funding Statement: Not Applicable

Conflict of Interest: The authors declare that they have no conflicts of interest.

Author contributions: All the authors have contributed in numerical calculation, framing, and writing the manuscript.

Availability of data and material: The research data of this manuscript will not be available.

Compliance with ethical standards: This article does not contain any studies involving animals or human participants performed by any of the authors.

Consent to participate: Not Applicable

Consent for Publication: Yes

Acknowledgments: Authors would like to thank Graphic Era (Deemed to be University) for their support and permission to communicate this research paper.

\section{References}

[1] D. J. Frank, R. H. Dennard, E. Nowak, P. M. Solomon, Y. Taur, and H. S. P. Wong, "Device scaling limits of Si MOSFETs and their application dependencies," Proc. IEEE, vol. 89, no. 3, pp. 259-287, 2001.

[2] K. Roy, S. Mukhopadhyay, and H. Mahmoodi-Meimand, "Leakage current mechanisms and leakage reduction techniques in deep-submicrometer CMOS circuits," Proc. IEEE, vol. 91, no. 2, pp. 305-327, 2003. 
[3] V. Mishra, Y. Kumar, V. Prateek, K. Verma, and S. Kumar, "EMA-based modeling of the surface potential and drain current of dual-material gate-all-around TFETs," J. Comput. Electron., vol. 17, no. 4, pp. 1596$1602,2018$.

[4] J. Wu, S. Member, and Y. Taur, "Reduction of TFET OFF -Current and Subthreshold Swing by Lightly Doped Drain," vol. 63, no. 8, pp. 3342-3345, 2016.

[5] K. Boucart and A. M. Ionescu, “Double-Gate Tunnel FET With High- k Gate Dielectric," vol. 54, no. 7, pp. 1725-1733, 2007.

[6] Z. Yang, "Tunnel Field-Effect Transistor with an L-Shaped Gate," IEEE Electron Device Lett., vol. 37, no. 7, pp. 839-842, 2016.

[7] S. K. Gupta and S. Kumar, "Analytical Modeling of a Triple Material Double Gate TFET with HeteroDielectric Gate Stack," 2018.

[8] K. H. Kao, A. S. Verhulst, W. G. Vandenberghe, B. Soree, G. Groeseneken, and K. De Meyer, "Direct and indirect band-to-band tunneling in germanium-based TFETs," IEEE Trans. Electron Devices, vol. 59, no. 2, pp. 292-301, 2012.

[9] Y. Zhao, S. Member, C. Wu, and S. Member, "Bandgap Engineering With Constant Sub-Threshold Slope Over 5 Decades of Current and High I ON / I OFF Ratio," vol. 38, no. 5, pp. 540-543, 2017.

[10] B. R. Raad, D. Sharma, P. Kondekar, K. Nigam, and D. S. Yadav, “Drain Work Function Engineered Doping-Less Charge Plasma TFET for Ambipolar Suppression and RF Performance Improvement: A Proposal, Design, and Investigation," IEEE Trans. Electron Devices, vol. 63, no. 10, pp. 3950-3957, 2016.

[11] S. Salahuddin, "Use of Negative Capacitance to Provide Voltage Amplification for Low Power Nanoscale Devices," Nano Lett, 2008.

[12] V. Mishra, Y. K. Verma, and S. K. Gupta, "Surface potential-based analysis of ferroelectric dual material gate all around (FE-DMGAA) TFETs," Int. J. Numer. Model. Electron. Networks, Devices Fields, vol. 33 , no. 4, pp. 1-11, 2020.

[13] P.-Y. Wang and B.-Y. Tsui, " $\$ \$ \$ r m S i \$ \$ \$ \$ \$ r m x \$$

$\{\$ \$ \backslash$ rm Ge\$ $\}$ _ $\$\{\$ 1 \$ \backslash$ hbox $\$\{\$-\$\}$

\section{$\$ \$ \$ r m x \$$}

\}\$ \$ Epitaxial Tunnel Layer Structure for P-Channel Tunnel FET Improvement," IEEE Trans. Electron Devices, vol. 60, no. 12, pp. 4098-4104, 2013. 
[14] J. P. Colinge et al., "Nanowire transistors without junctions," Nat. Nanotechnol., vol. 5, no. 3, pp. 225229, 2010.

[15] G. Leung and C. O. Chui, "Variability impact of random dopant fluctuation on nanoscale junctionless FinFETs," IEEE Electron Device Lett., vol. 33, no. 6, pp. 767-769, 2012.

[16] R. J. E. Hueting, B. Rajasekharan, C. Salm, and J. Schmitz, "The charge plasma P-N diode," IEEE Electron Device Lett., vol. 29, no. 12, pp. 1367-1369, 2008.

[17] A. K. Jain, S. Sahay, and M. J. Kumar, "Controlling L-BTBT in Emerging Nanotube FETs Using DualMaterial Gate," IEEE J. Electron Devices Soc., vol. 6, no. January, pp. 611-621, 2018.

[18] Z. X. Chen et al., "Demonstration of tunneling FETs based on highly scalable vertical silicon nanowires," IEEE Electron Device Lett., vol. 30, no. 7, pp. 754-756, 2009.

[19] K. P. Pradhan, S. K. Mohapatra, P. K. Sahu, and D. K. Behera, "Impact of high-k gate dielectric on analog and RF performance of nanoscale DG-MOSFET," Microelectronics J., vol. 45, no. 2, pp. 144-151, 2014.

[20] V. Mishra, Y. Verma Kumar, and S. Kumar Gupta, "Investigation of Localized Charges on Linearity and Distortion Performance of Ferroelectric Dual Material Gate All Around TFETs," J. NANO-Electron. Phys., vol. 11, no. 4, pp. 1-6, 2019.

\section{Figures}

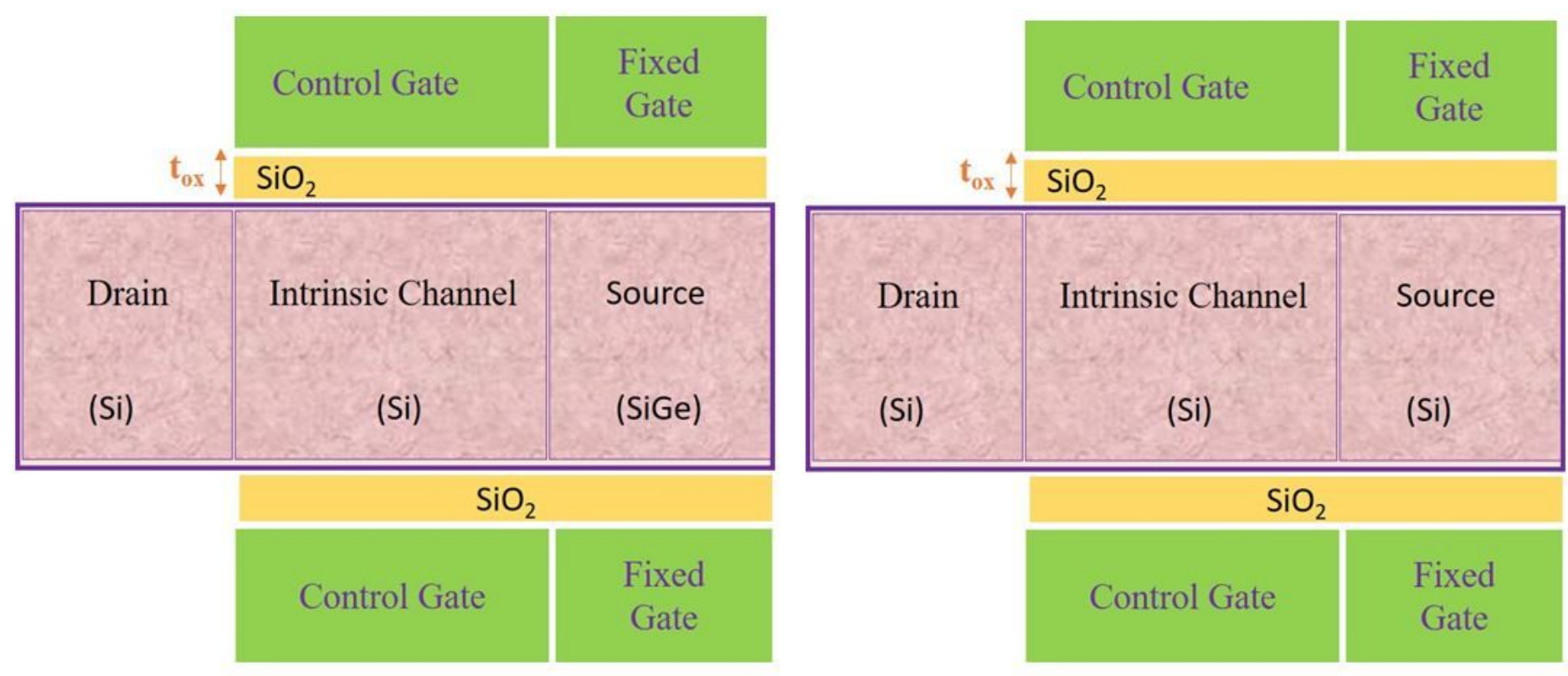

\section{Figure 1}


2-D structures of DL-DG-TFET with (a) Si0.5Ge0.5 source, and (b) Si source.

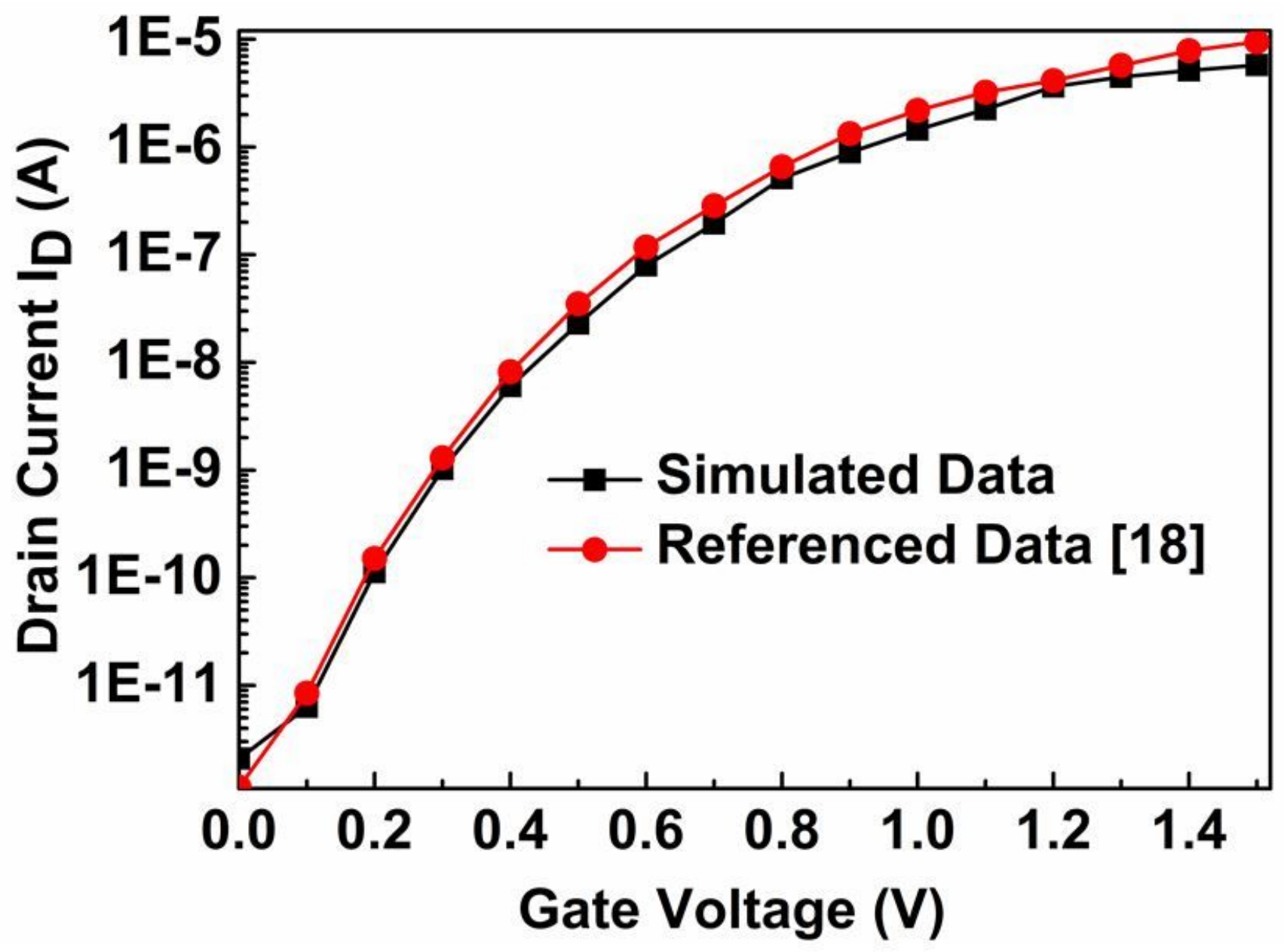

Figure 2

Validation curve with the published experimental work [18].
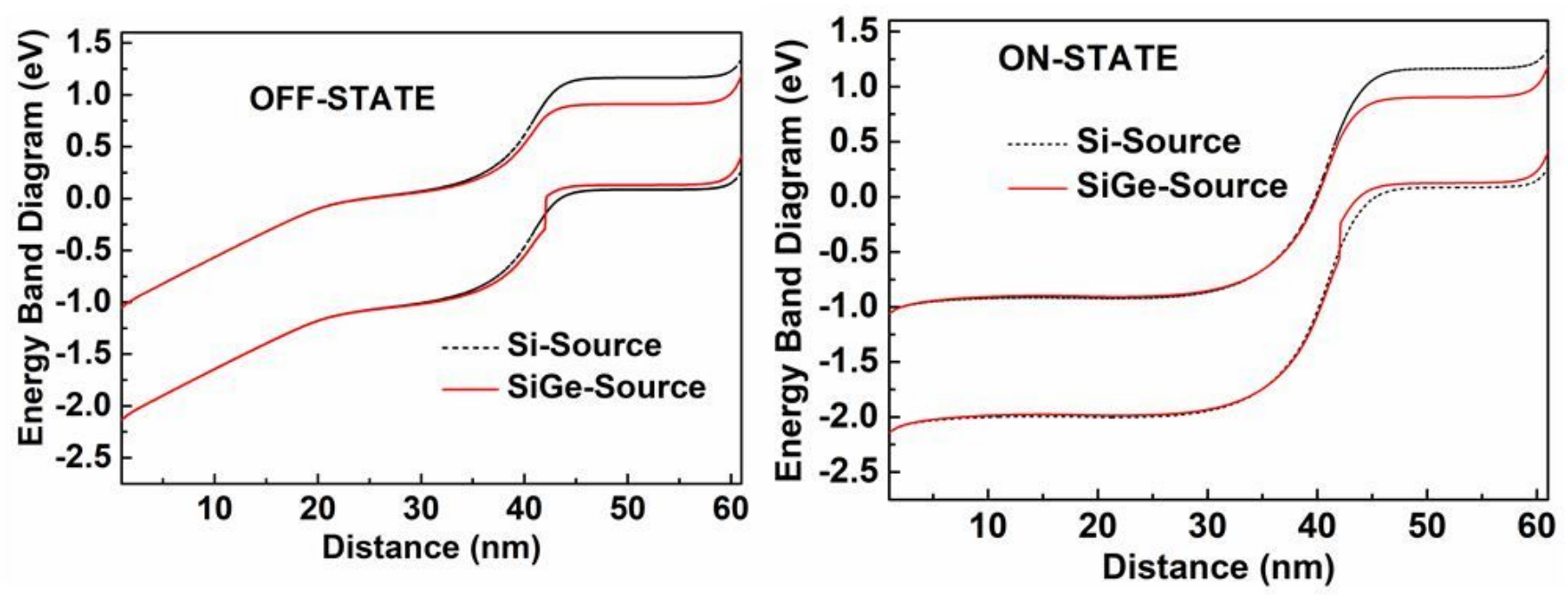
Energy band diagram of DL-DG-TFET with Si0.5Ge0.5 source and Si source in (a) OFF-state, and (b) ONstate.

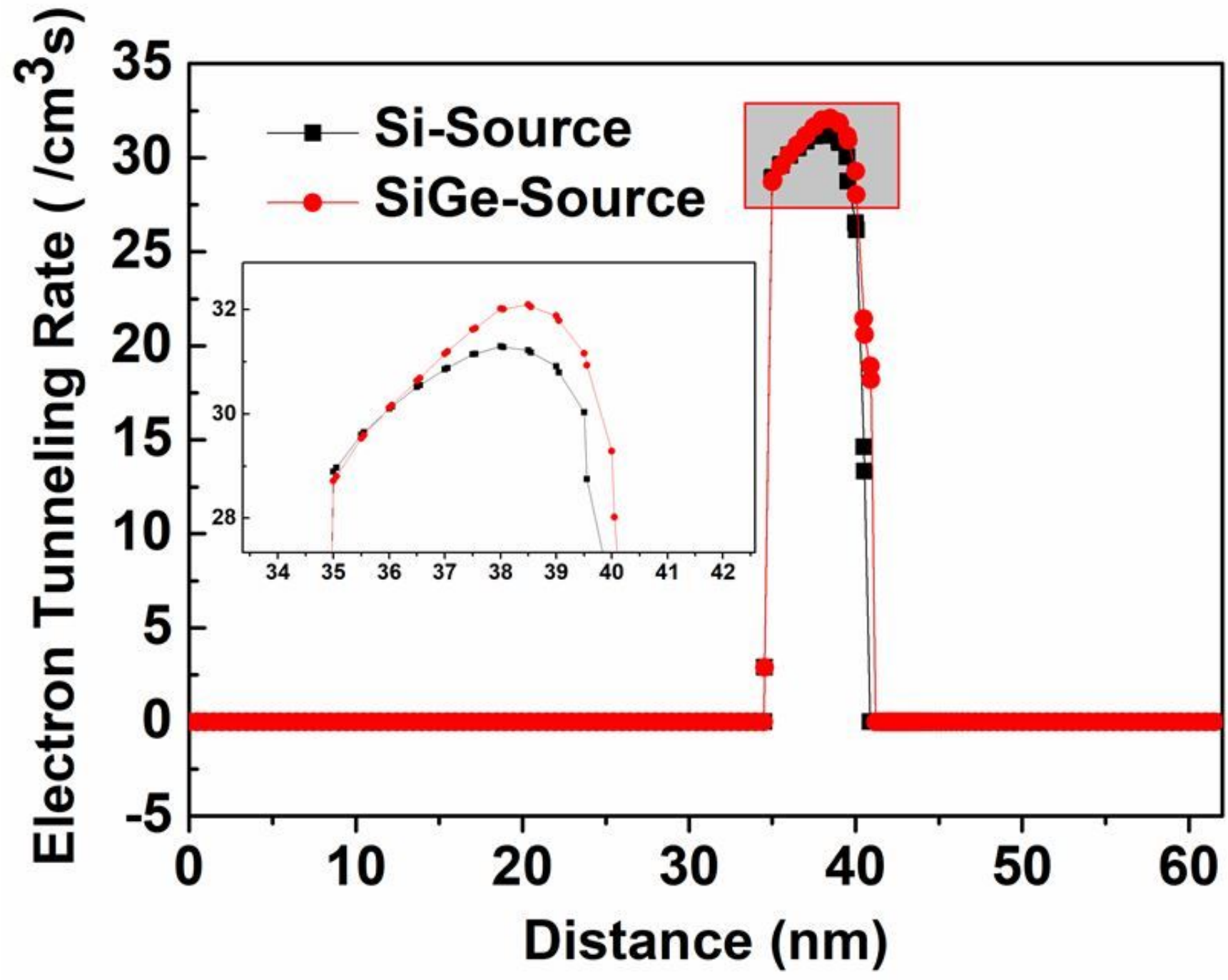

Figure 4

BTBT rate of DL-DG-TFET with Si0.5Ge0.5 source and Si source at VGS $=1 \mathrm{~V}$ and VDS $=1 \mathrm{~V}$. 


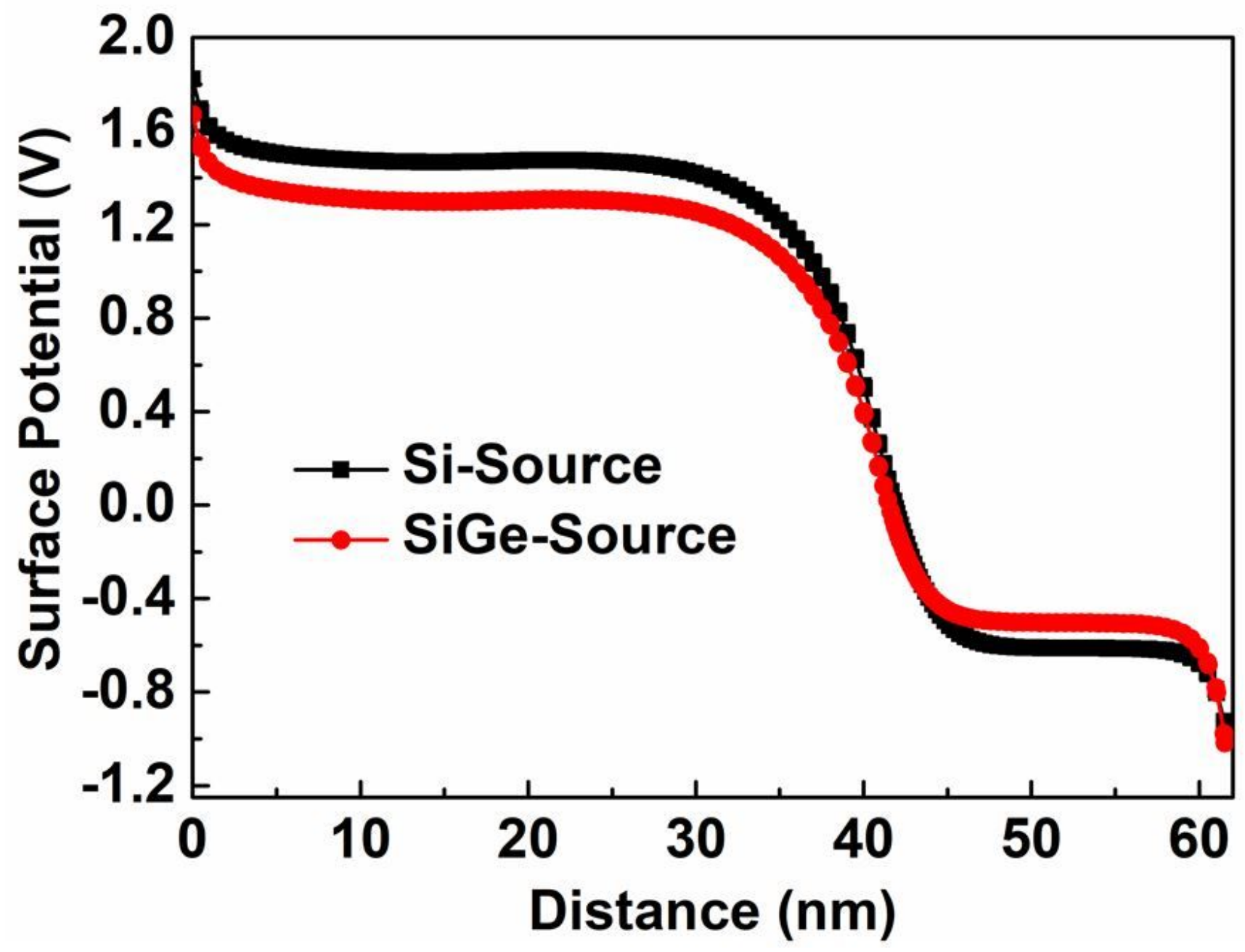

Figure 5

Surface potential variation of DL-DG-TFET with Si0.5Ge0.5 source and Si source at VGS $=1 \mathrm{~V}$ and VDS $=$ $1 \mathrm{~V}$. 


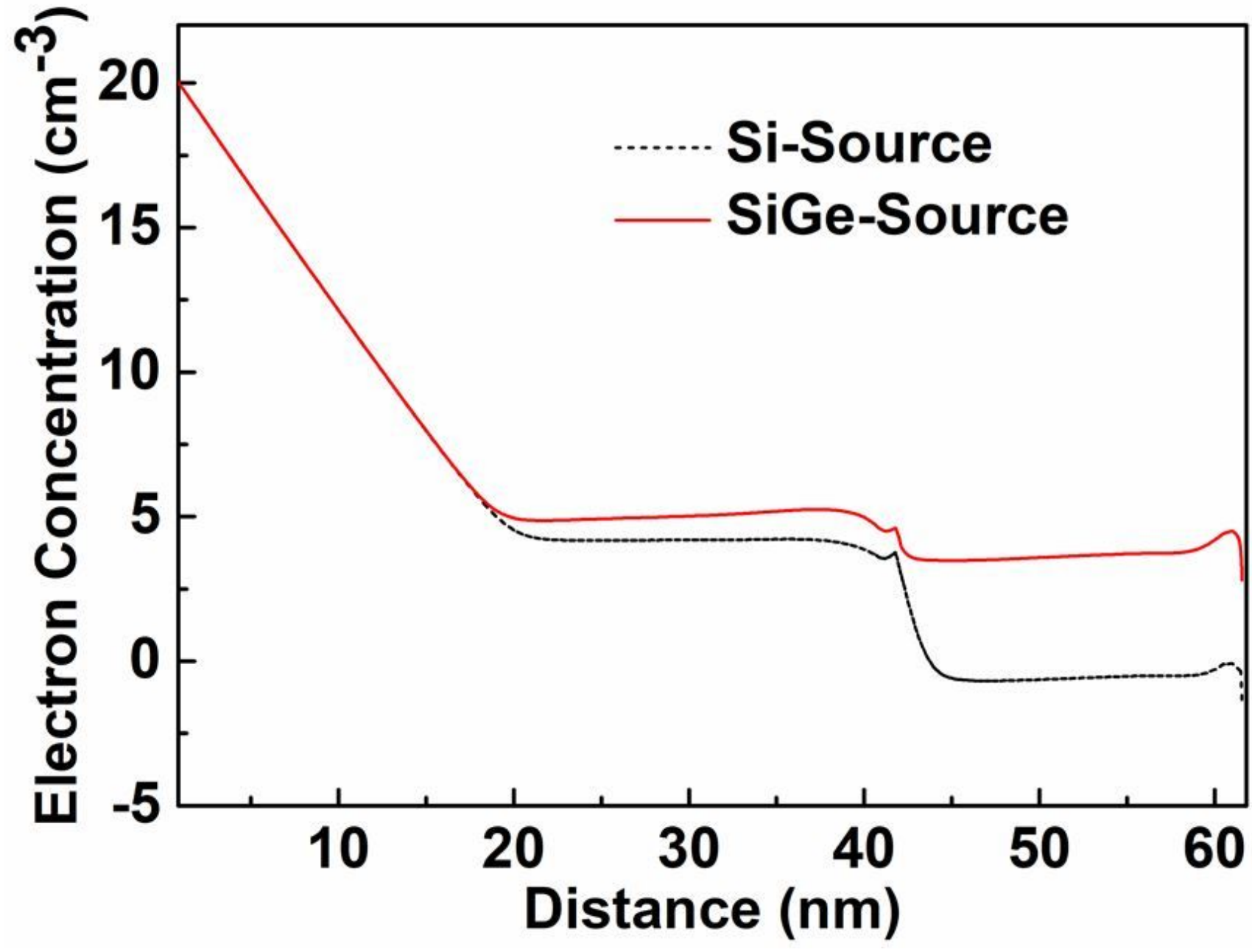

Figure 6

Electron concentration variation of DL-DG-TFET with Si0.5Ge0.5 source and Si source at VGS $=0 \mathrm{~V}$ and VDS $=1$ 


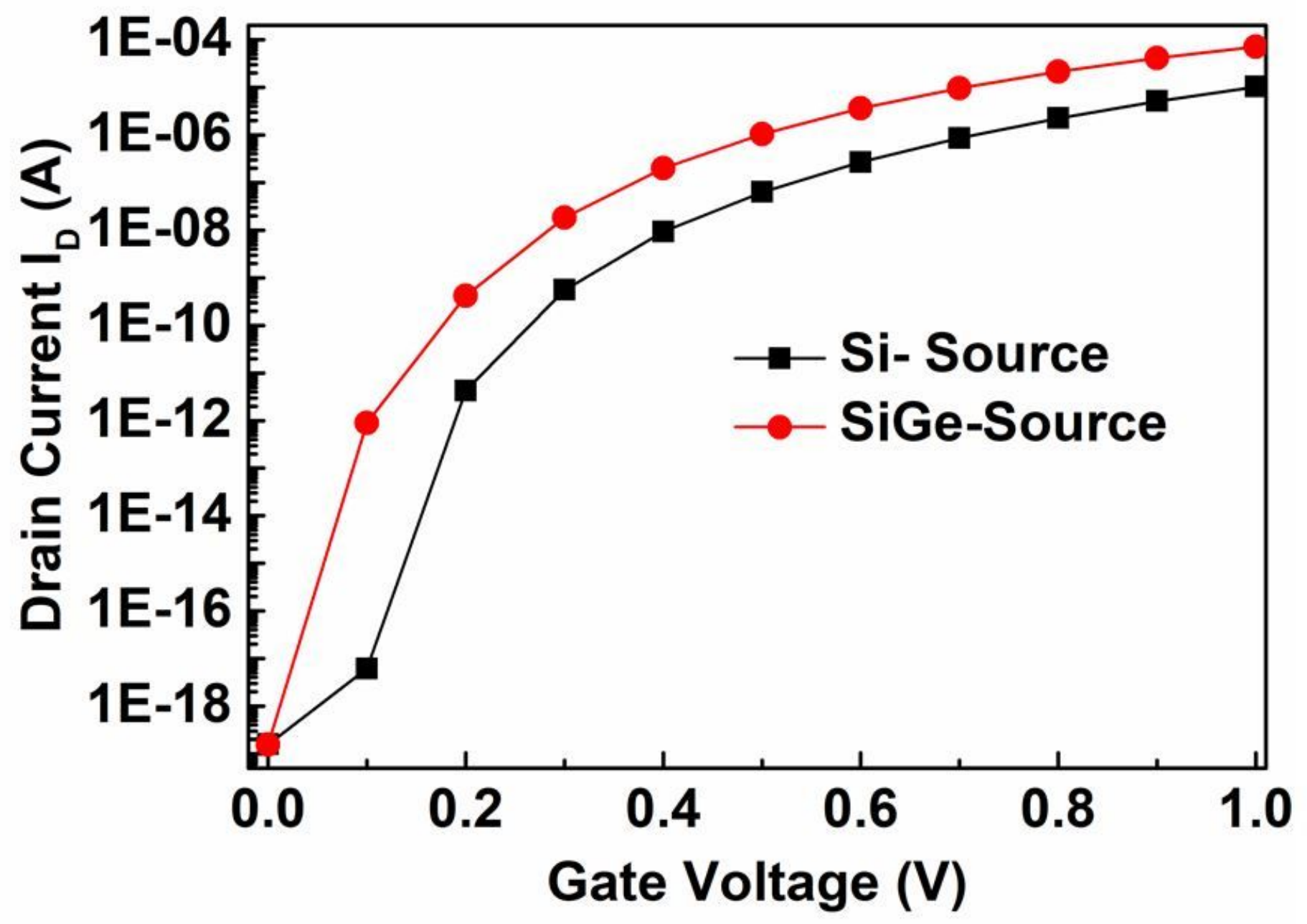

Figure 7

ID-VGS variation of DL-DG-TFET with Si $0.5 \mathrm{Ge} 0.5$ source and Si source at VDS $=1 \mathrm{~V}$. 


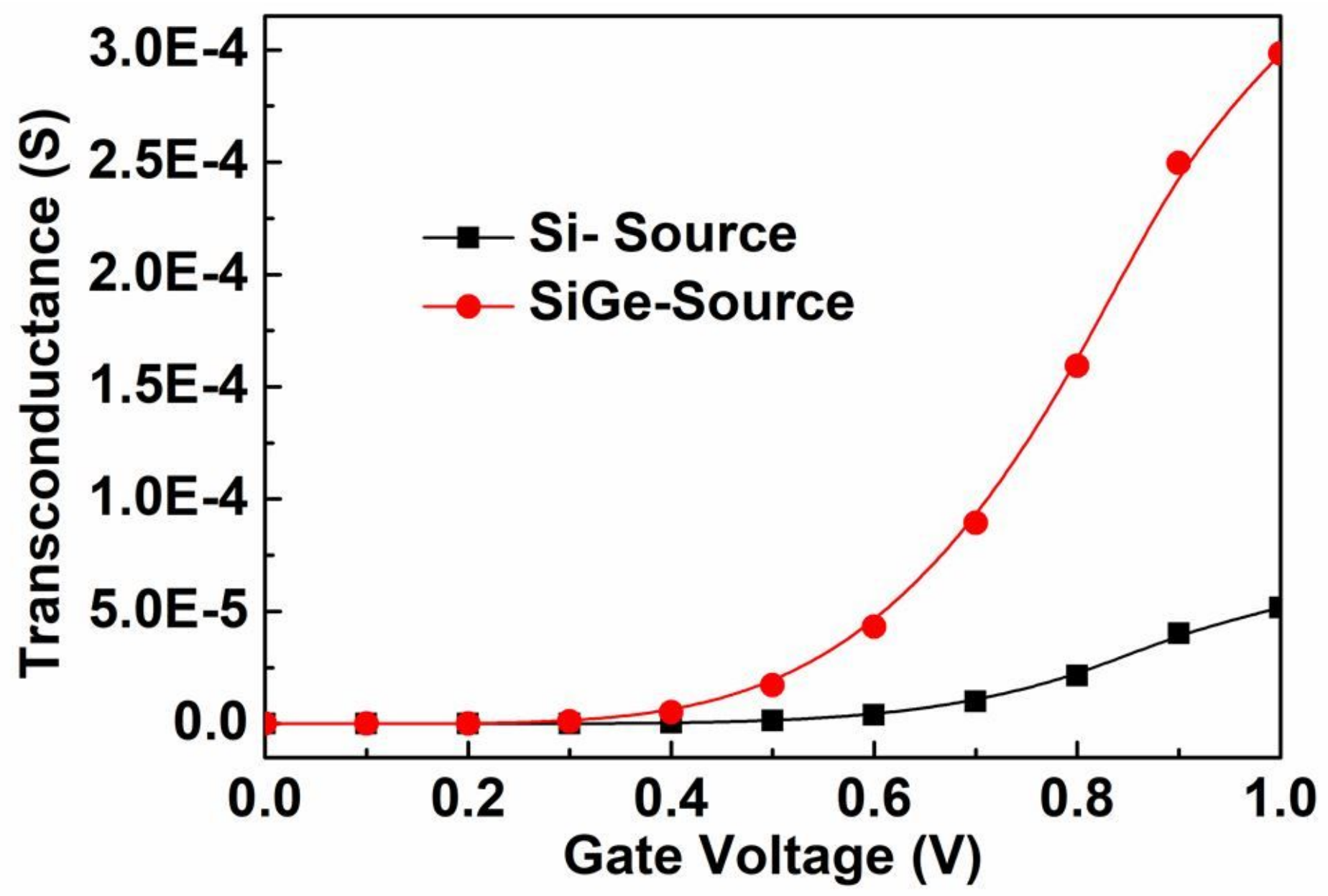

Figure 8

Transconductance variation of DL-DG-TFET with Si0.5Ge0.5 source and Si source at VDS $=1 \mathrm{~V}$.
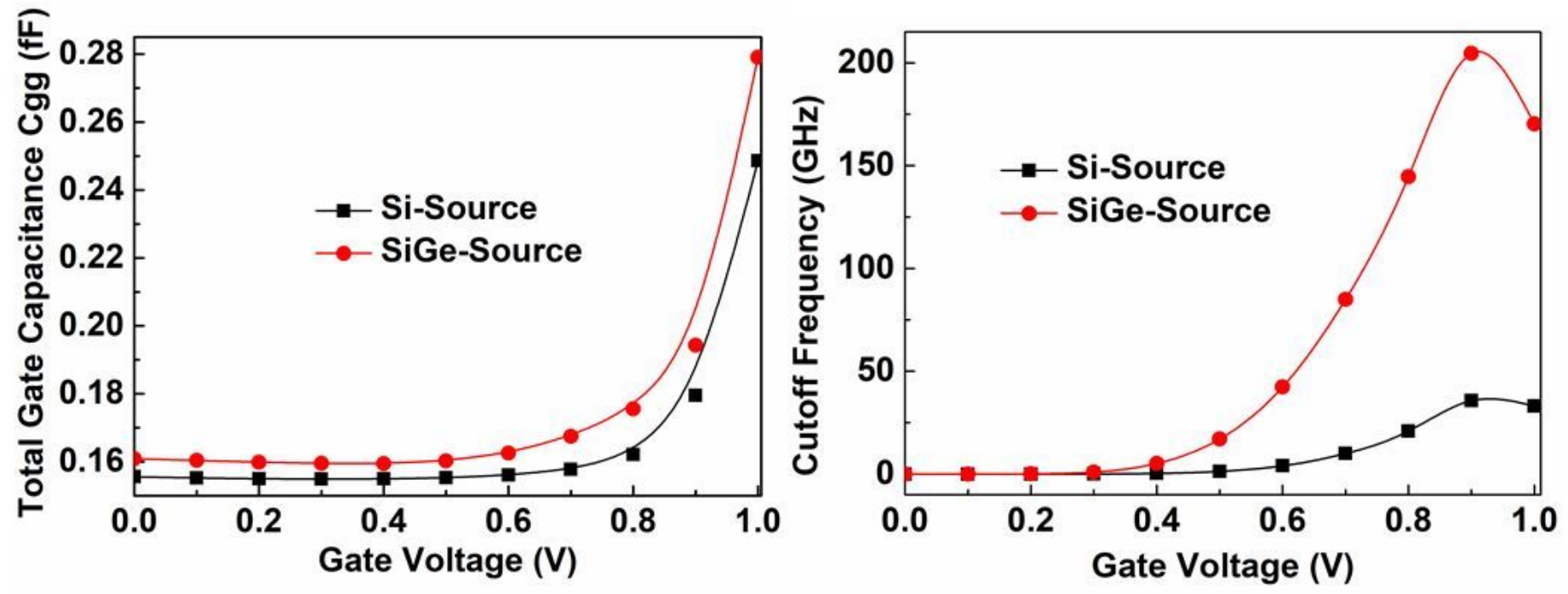

Figure 9 
Comparison of DL-DG-TFET with Si0.5Ge0.5 source and Si source in terms of (a) Total gate capacitance, and (b) Cut-off frequency.

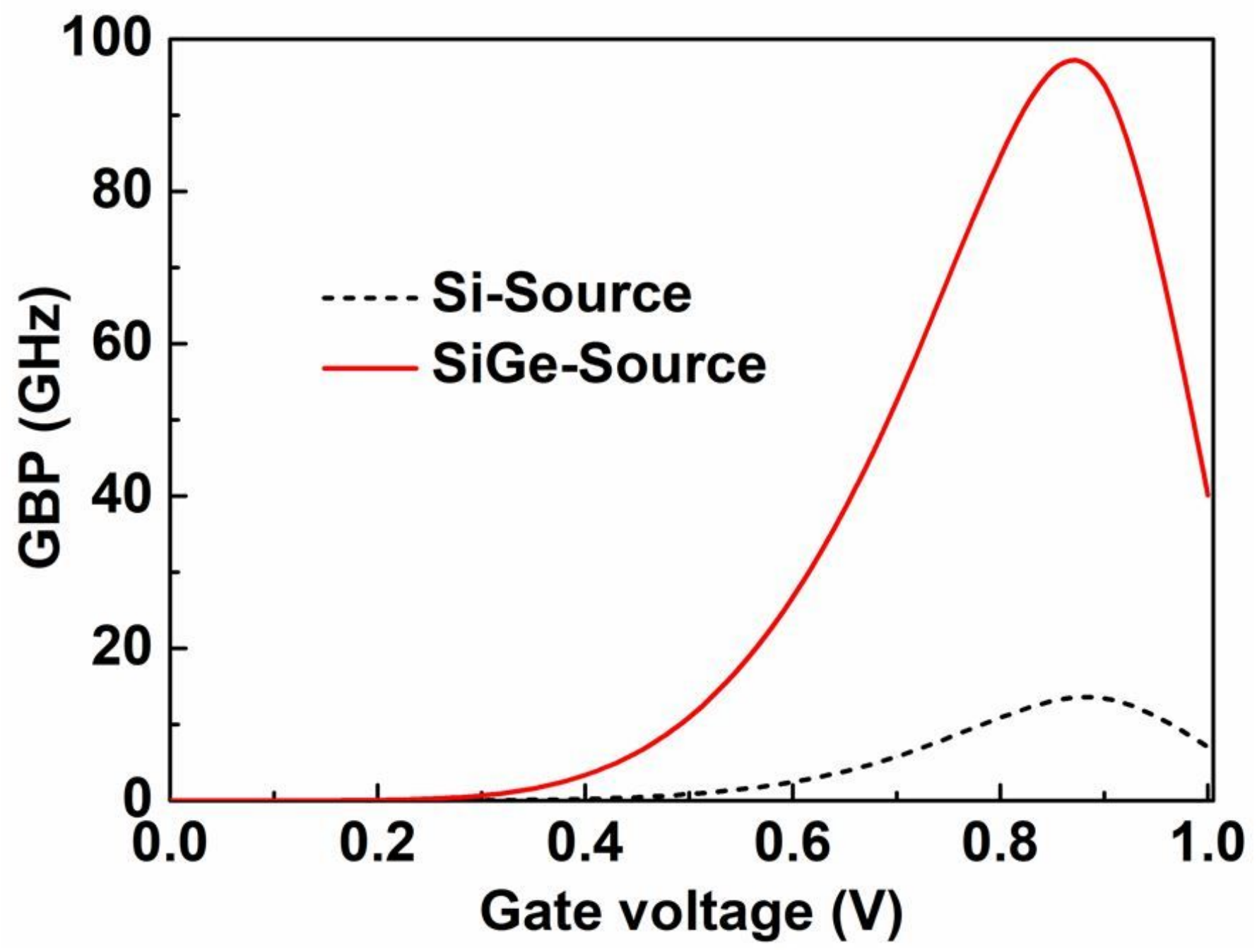

Figure 10

GBP variation of DL-DG-TFET with Si0.5Ge0.5 source and Si source. 


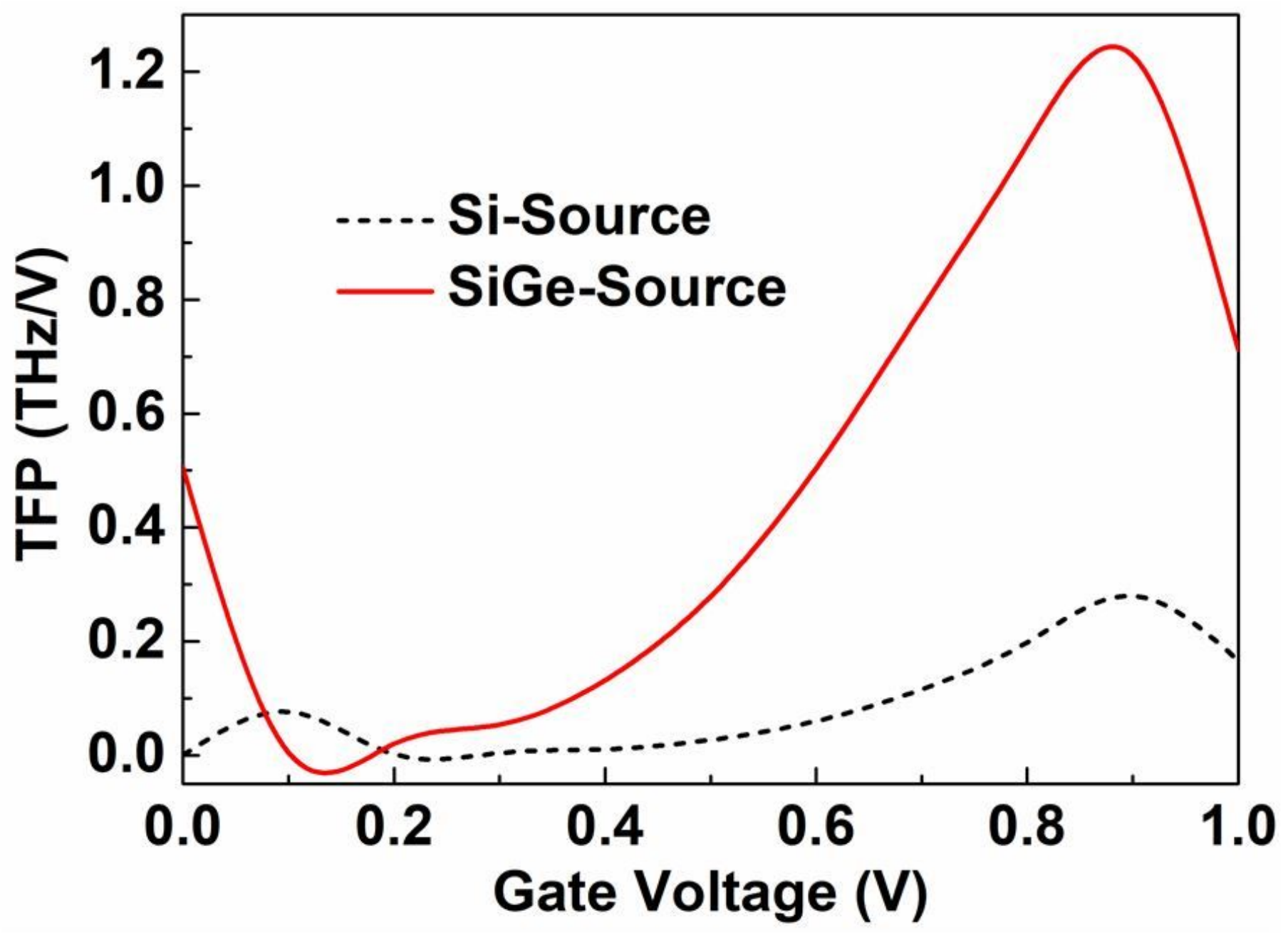

Figure 11

TFP variation of DL-DG-TFET with Si0.5Ge0.5 source and Si source.
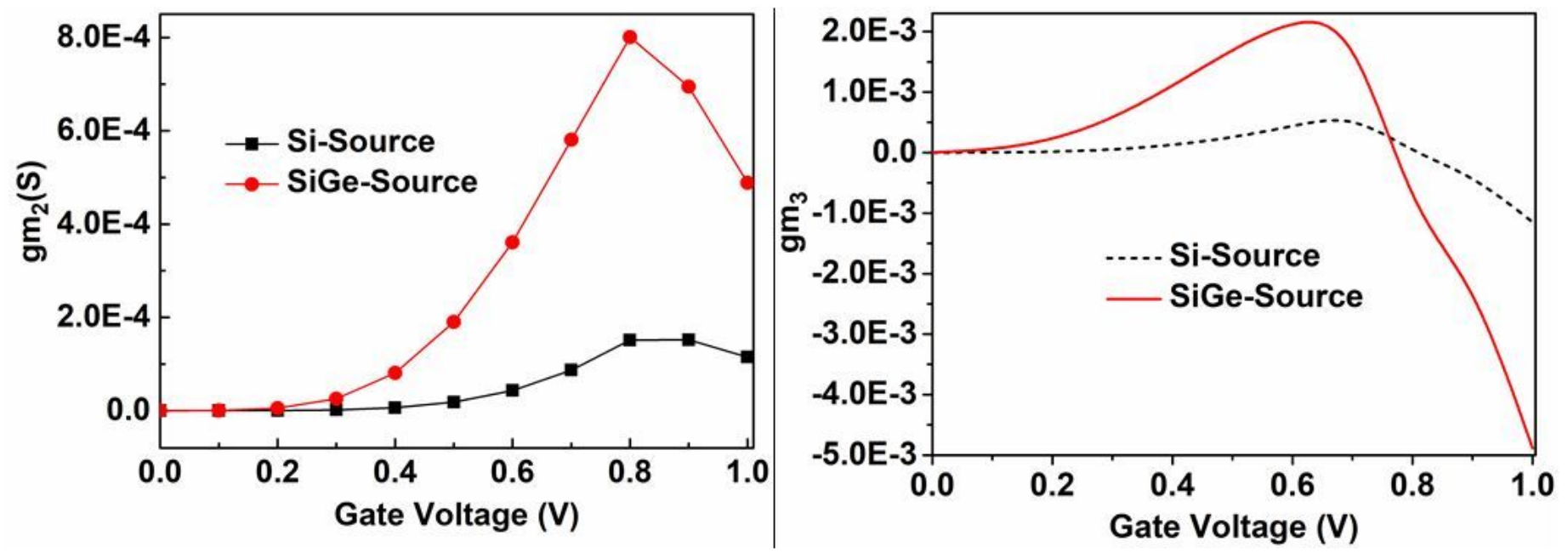

Figure 12 
Comparison of DL-DG-TFET with Si0.5Ge0.5 source and Si source in terms of (a) gm2, and (b) gm3.
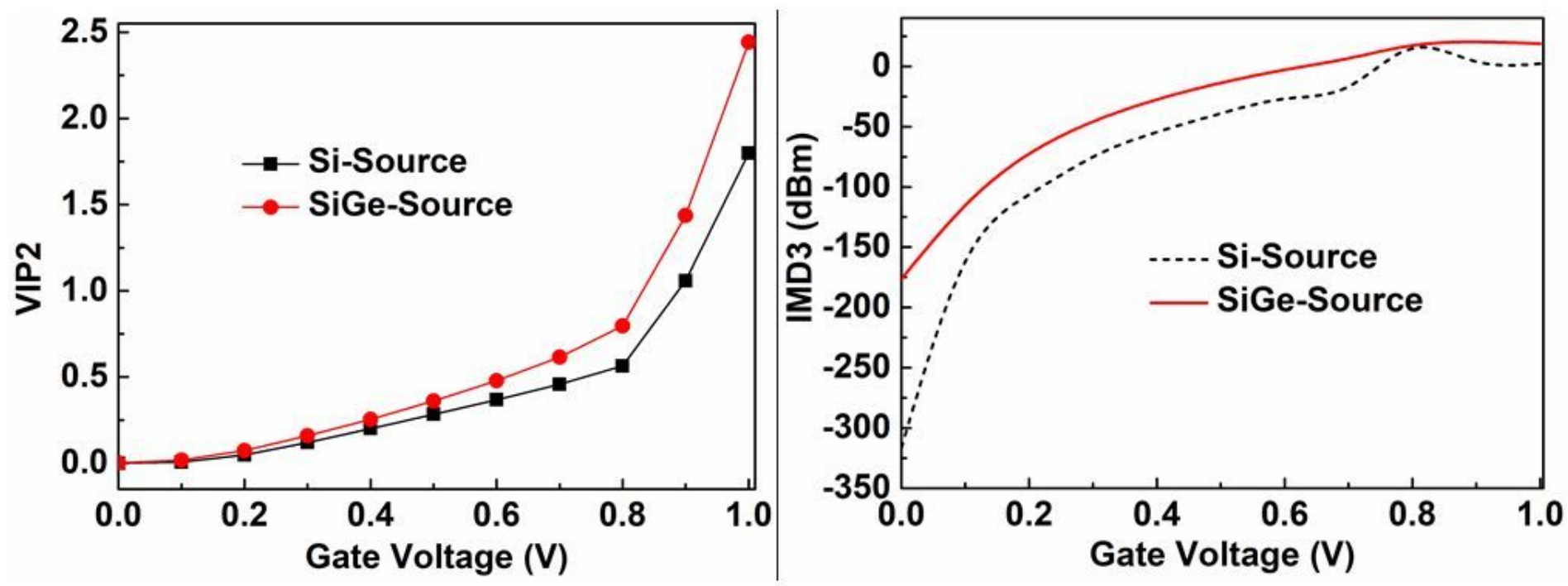

Figure 13

Comparison of DL-DG-TFET with Si0.5Ge0.5 source and Si source in terms of (a) VIP2, and (b) IMD3.

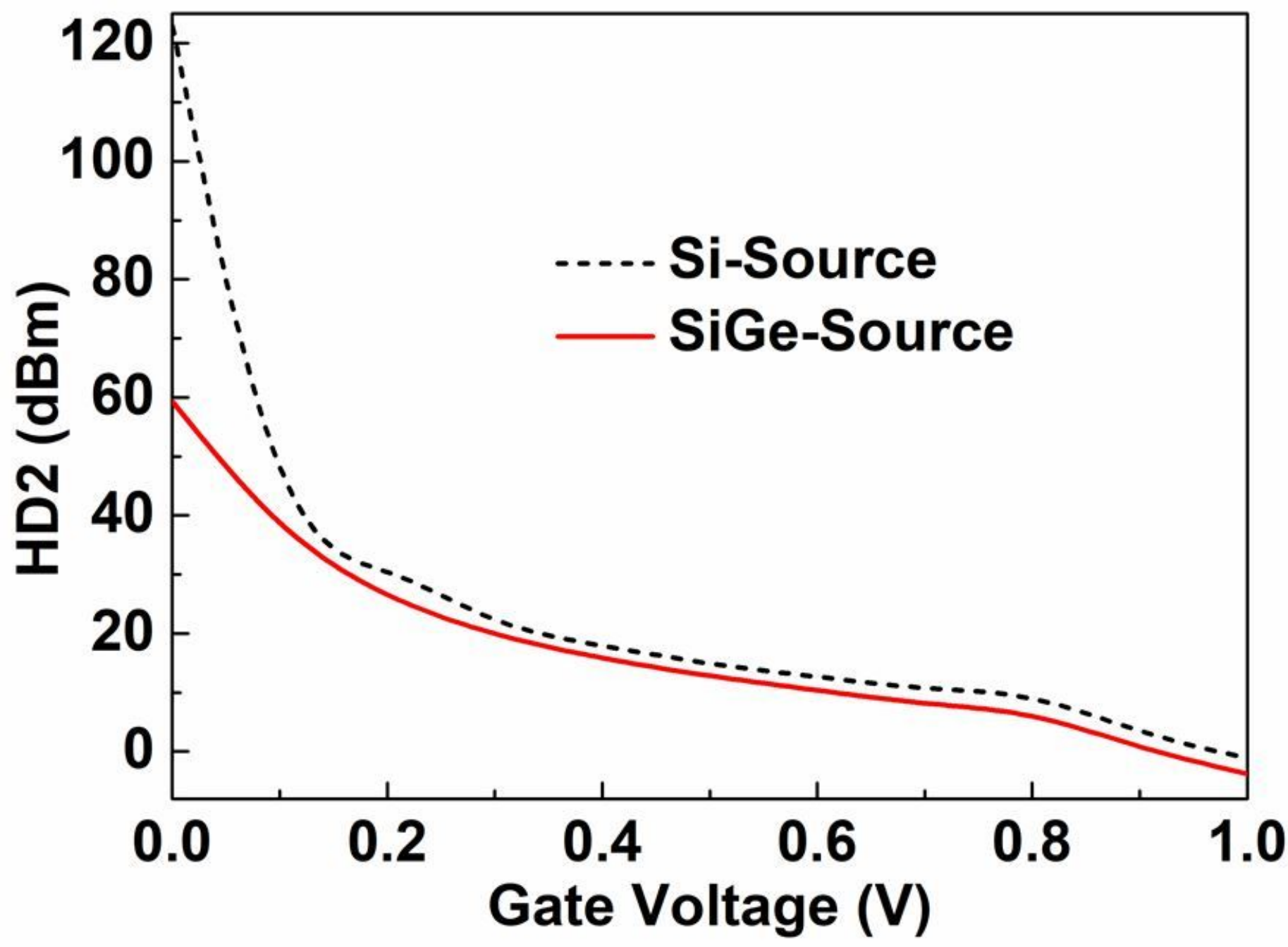


HD2 variation of DL-DG-TFET with Si0.5Ge0.5 source and Si source 